\title{
Frecuencia de consumo de alimentos en adolescentes y adultos jóvenes con síndrome de Down, octubre - 2017
}

Mayra Guisela Barco Díaz* 1,a

RESUMEN

Objetivo: Describir la frecuencia de consumo de alimentos en adolescentes y adultos jóvenes con síndrome de Down de tres centros de talleres privados.

Materiales y métodos: Se aplicó un cuestionario de frecuencia de consumo de alimentos a los padres para conocer sus hábitos alimentarios.

Resultados: El 55 \% de los encuestados consumió lácteos diariamente, y $60 \%$ ingirió carnes magras de 3 a 4 veces por semana. Las legumbres se consumieron de 3 a 4 veces por semana por $75 \%$ de los participantes. El $5 \%$ consumió pescado de 4 a más veces por semana. El $15 \%$ ingirió huevo 3 veces por semana; $45 \%$, frutas diariamente; y $25 \%$, verduras y hortalizas todos los días. El $100 \%$ consumió bebidas azucaradas diariamente. Semanalmente, $45 \%$ ingirió pasteles o galletas; $40 \%$, mantequilla o margarina; $30 \%$, embutidos; y $40 \%$, golosinas.

Conclusiones: Más de la mitad de los participantes consumió adecuadamente lácteos, carnes magras y legumbres pero no pescado, huevo, verduras y hortalizas. Las bebidas azucaradas, pasteles o galletas, mantequilla o margarina, embutidos y golosinas son ingeridos de forma inapropiada. La ingesta de frutas, en relación a la variedad y la forma de consumirlas, es también inadecuada.

Palabras clave: Síndrome de Down; Hábitos alimentarios; Perú (Fuente : DeCS BIREME).

\section{Frequency of food consumption in teens and young adults with Down syndrome - October 2017}

\section{ABSTRACT}

Objective: To describe the frequency of food consumption in teens and young adults with Down syndrome from three private workshops.

Materials and methods: A questionnaire on the frequency of food consumption was administered to the study population's parents to find out their children's food habits.

Results: Fifty-five percent (55\%) of the study population consumed dairy products on a daily basis, $60 \%$ lean meat 3 to 4 times per week, $75 \%$ legumes 3 to 4 times per week, $5 \%$ fish 4 times or more per week, $15 \%$ eggs 3 times per week, $45 \%$ fruits daily, $25 \%$ greens and vegetables every day, $100 \%$ sweetened beverages daily, $45 \%$ cakes or cookies during the week, $40 \%$ butter or margarine during the week, $30 \%$ sausages and lunch meats during the week, and $40 \%$ candies during the week.

Conclusions: More than half of the participants had an adequate consumption of dairy products, lean meat and legumes, but an inadequate consumption of fish, eggs, and greens and vegetables. Furthermore, the consumption of sweetened beverages, cakes or cookies, butter or margarine, sausages and lunch meats, and candies was inadequate. Fruit variety and mode of consumption were also inadequate.

Keywords: Down syndrome; Feeding behavior; Peru (Source: MeSH NLM).

1. Universidad Nacional Mayor de San Marcos, Facultad de Medicina. Lima, Perú.

a. Maestranda en Nutrición con Mención Clínica, Licenciada en Nutrición.

*Autor corresponsal 


\section{INTRODUCCIÓN}

Según la Organización Mundial de la Salud, la incidencia estimada de síndrome de Down (SD) es 1 de cada 1100 recién nacidos en todo el mundo (1). En el Perú, el Registro Nacional de Personas con Discapacidad cargo del Consejo Nacional para la Integración de la Persona con Discapacidad (CONADIS) al 2015 tiene inscritas 8800 personas con SD ${ }^{(2)}$.

El síndrome de Down (SD) es el cuadro clínico que deriva de la presencia de 47 cromosomas en el núcleo de las células de un organismo humano, en lugar de los 46 que existen normalmente. Entre las características generales del fenotipo SD se encuentra discapacidad cognitiva, hipotonía muscular, hiperlaxitud ligamentaria, hiporreflexia, facies peculiar ${ }^{(3)}$.

Cuando nace con el SD, el niño tiene bajo peso. Cerca al primer mes de vida alcanza el nivel adecuado, o empieza a recuperarlo; luego, el peso se incrementa a parámetros esperados para las características del niño. Después del primer año de vida, hay una tendencia a la obesidad, que se eleva con la edad y se asocia a enfermedades que disminuyen la esperanza de vida de esta población (4). Diversos estudios han demostrado que la prevalencia de obesidad es mayor que en la población en general, aunque no se conocen los mecanismos que la explican, algunos estudios sugieren factores como alta prevalencia de hipotiroidismo, tasa metabolica basal más baja, alimentacion inadecuada, bajo nivel de actividad física y genética ${ }^{(5)}$.

Otra característica de las personas con SD es un retraso variable en la adquisición del reflejo faríngeo de la deglución, lo que origina, con frecuencia, atragantamientos e, incluso, aspiración de alimentos o líquidos. Asimismo, la hipertrofia adenoidea o amigdalar y la hipotonía lingual dificultan más el mecanismo deglutorio, pues obligan a los niños a mantener la respiración bucal mientras ingieren alimentos. Por otro lado, los problemas de motilidad esofágica son más frecuentes en las personas con síndrome de Down (adultos o niños) que en la población general. La suma de estas situaciones genera frecuentes problemas de deglución en las personas con síndrome de Down a lo largo de toda su vida ${ }^{(6)}$.

Los problemas de masticación y alimentación se presentan en distintas etapas de la vida, desde la primera infancia hasta la adultez. Las características anatómicas y funcionales del SD repercuten de forma directa sobre la salud oral, ya que provocan la disfunción orofacial debido al pobre control neuromotor, las anomalías dentales, la dismorfología facial y las enfermedades intercurrentes ${ }^{(7)}$.

En el Perú existen muy pocos trabajos publicados sobre el estado nutricional y prácticas alimentarias en adolescentes y adultos jóvenes con SD. Gómez y Gutiérrez realizaron un estudio en la ciudad de Lima, en adolescentes con SD entre 15 a 20 años, en el que se estimó la energía consumida mediante la frecuencia de consumo de alimentos ${ }^{(8)}$. Charca evaluó el estado nutricional y el consumo de alimentos de escolares con SD entre 6 y 16 años de la ciudad de Puno ${ }^{(9)}$.

El objetivo del presente estudio es describir el consumo de alimentos, y la variedad de consumo de frutas en adolescentes y adultos jóvenes miembros de la Sociedad Peruana SD, que acuden a tres centros de talleres educativos privados ubicados en la ciudad de Lima.

\section{MATERIALES Y MÉTODOS}

\section{Diseño y población del estudio}

El tipo de estudio fue descriptivo y transversal. La población estuvo conformada por 40 adolescentes y adultos jóvenes entre 16 y 29 años, miembros de la Sociedad Peruana de Síndrome Down, que a su vez acudían a 3 centros de talleres educativos privados ubicados en la ciudad de Lima. Se escogieron estos centros para la investigación porque a ellos asisten adolescentes y adultos jóvenes con SD. Entre los criterios de inclusión de los participantes que se tomó está el pertenecer a un nivel socioeconómico alto según la escala de Graffar modificada ${ }^{(10)}$. Este instrumento clasifica a la población según su nivel socioeconómico y evalúa 4 aspectos: nivel de instrucción y actividad laboral del jefe del hogar, grado de instrucción de la esposa del jefe del hogar y las características de la vivienda (material predominante de esta y saneamiento con que cuenta). Se consideró este criterio para asegurar que los participantes tenga el nivel socioeconómico suficiente para la adquisición de alimentos.

La muestra estuvo conformada por la totalidad de la población. Se realizaron las coordinaciones con las instituciones y luego se procedió a la captación de la información, se aplicó el cuestionario de frecuencia ${ }^{(11)}$ de consumo de alimentos a los padres de los participantes .

\section{Variables y mediciones}

Las variables fueron consumo de alimentos, la variedad del consumo de frutas y la forma como consumirla. En la variable consumo de alimentos, las dimensiones fueron cereales, fideos y tubérculos, carne magras, pescado, huevo, legumbres, lácteos, verduras y hortalizas, 
bebidas azucaradas, pasteles o galletas, margarina o mantequilla, embutidos y golosinas. Las dimensiones para la variedad consumo de frutas fueron jugo de frutas y frutas en estado natural de las siguientes plátano, papaya, mandarina, naranja, granadilla, melocotón, manzana, sandia, piña, pera y pasas. Los indicadores para todas las variables fueron las frecuencias de consumo de alimentos por semana.

Para la identificación de consumo de alimentos saludables,variedad de consumo de frutas y la forma como se consumió se aplicó el cuestionario Frecuencia de consumo de alimentos cualitatitva (FCAC) ${ }^{(11,12)}$, que fue elaborado por la autora para este estudio y validado por un juicio de expertas. La metodología utilizada para la elaboración de este instrumento se basó en un estudio realizado por el Ministerio de Salud del Perú que elaboró también un FCAC ${ }^{(13)}$.

Para determinar la frecuencia de consumo adecuado de alimentos se utilizaron las recomendaciones de la Federacion Española de Síndrome Down ${ }^{(14)}$, y la variedad de frutas y la manera de consumirlas se definieron con las recomendaciones de las Guías alimentarias para la población peruana ${ }^{(15)}$.

\section{Análisis estadísticos}

Los datos obtenidos fueron digitados en el programa Excel y se presentaron mediante las estadísticas descriptivas.

\section{Consideraciones éticas}

Se solicitó el consentimiento informado a los padres de los participantes y a los participantes su asentimiento.

\section{RESULTADOS}

Se evaluaron 40 personas con SD (24 hombres y 16 mujeres) entre 16 y 29 años. Al analizar la frecuencia del consumo de alimentos (Tabla 1), se observó que $55 \%$ de los participantes consumió lácteos diariamente. El $100 \%$ de los encuestados ingirió de 2 a 3 raciones de cereales, fideos o tubérculos al día; y 75 \% consumió la misma proporción de legumbres a la semana. Además, $45 \%$ consumió frutas y $25 \%$ verduras y hortalizas diariamente. En una semana, $5 \%$ ingirió de 4 a más raciones de pescado; mientras que $60 \%$, de 3 a 4 raciones de carne magra, y 15 \% consumió 3 unidades de huevo.

Tabla 1. Frecuencia de consumo de alimentos en personas con SD

\begin{tabular}{|c|c|c|c|c|c|c|c|c|}
\hline & \multicolumn{8}{|c|}{ Raciones a la semana } \\
\hline & Diario & 6 & 5 & 4 & 3 & 2 & 1 & $\begin{array}{c}\text { No } \\
\text { consume }\end{array}$ \\
\hline Verduras y hortalizas & $25 \%$ & $32,5 \%$ & & & $15 \%$ & $27,5 \%$ & & \\
\hline Frutas & $45 \%$ & $30 \%$ & $5 \%$ & & $15 \%$ & & & $5 \%$ \\
\hline Cerales, fideos, tubérculos & $100 \%$ & & & & & & & \\
\hline Carnes magras & $20 \%$ & $10 \%$ & $10 \%$ & $20 \%$ & $40 \%$ & & & \\
\hline Pescado & $5 \%$ & & & & $10 \%$ & $72,5 \%$ & & $12,5 \%$ \\
\hline Huevo & $25 \%$ & $45 \%$ & & & $15 \%$ & & & $15 \%$ \\
\hline Legumbres & & & $5 \%$ & $5 \%$ & $70 \%$ & $5 \%$ & & $15 \%$ \\
\hline Lácteos & $55 \%$ & & $10 \%$ & $10 \%$ & $15 \%$ & & & $10 \%$ \\
\hline
\end{tabular}

En relación a las frutas, el plátano fue el producto consumido con mayor frecuencia: $35 \%$, de 4 a más veces por semana; y $45 \%$, de 1 a 3 veces por semana. El $60 \%$ consumió jugo de frutas de 4 a más veces por semana.
Los productos con menor frecuencia de consumo fueron la granadilla ( 60 \% no la consumió ), naranja ( $65 \%$ ) y manzana ( 35 \% ) (Figura 1). 
Frecuencia de consumo de alimentos en adolescentes y adultos jóvenes con síndrome de Down, octubre - 2017

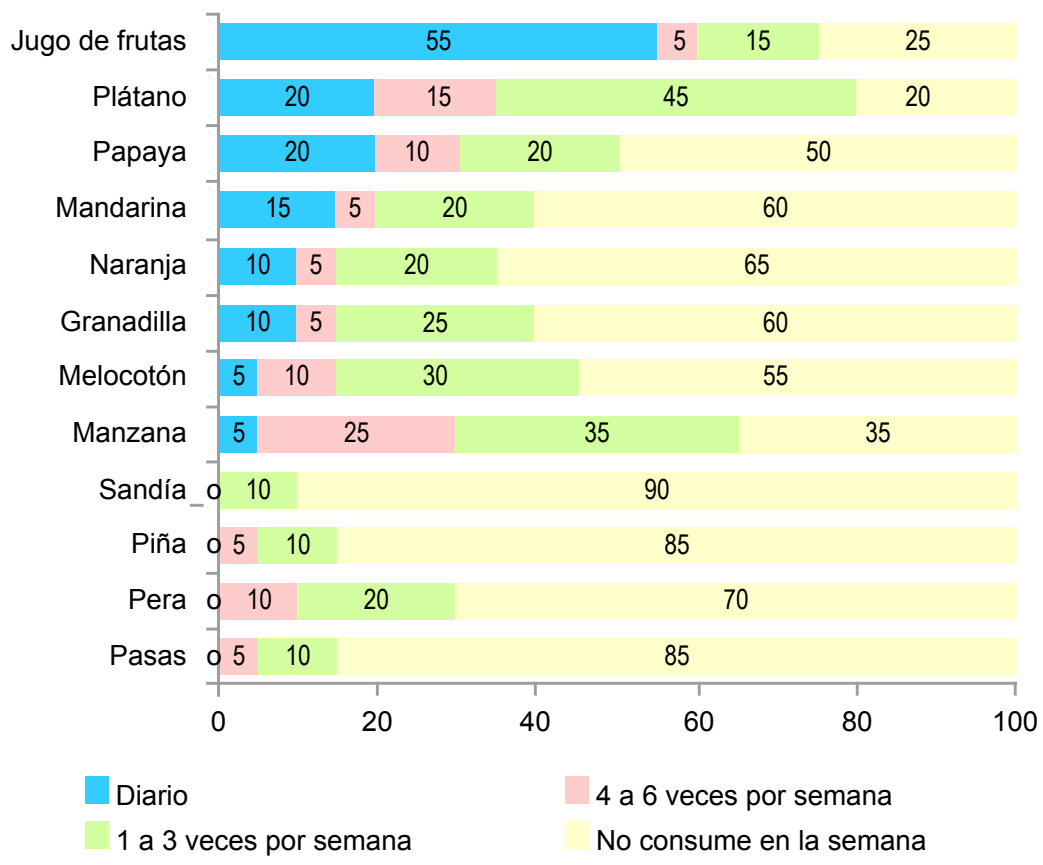

Figura 1. Frecuencia de consumo de frutas (entera y en jugo) en personas con SD

Respecto a a los alimentos con alto contenido calórico, $25 \%$ ingirió embutidos; $10 \%$, pasteles o galletas; el $15 \%$, frecuencia de 4 a 6 veces por semana. El $100 \%$ consumió mantequilla y / o margarina y $12,5 \%$, golosinas, todos con

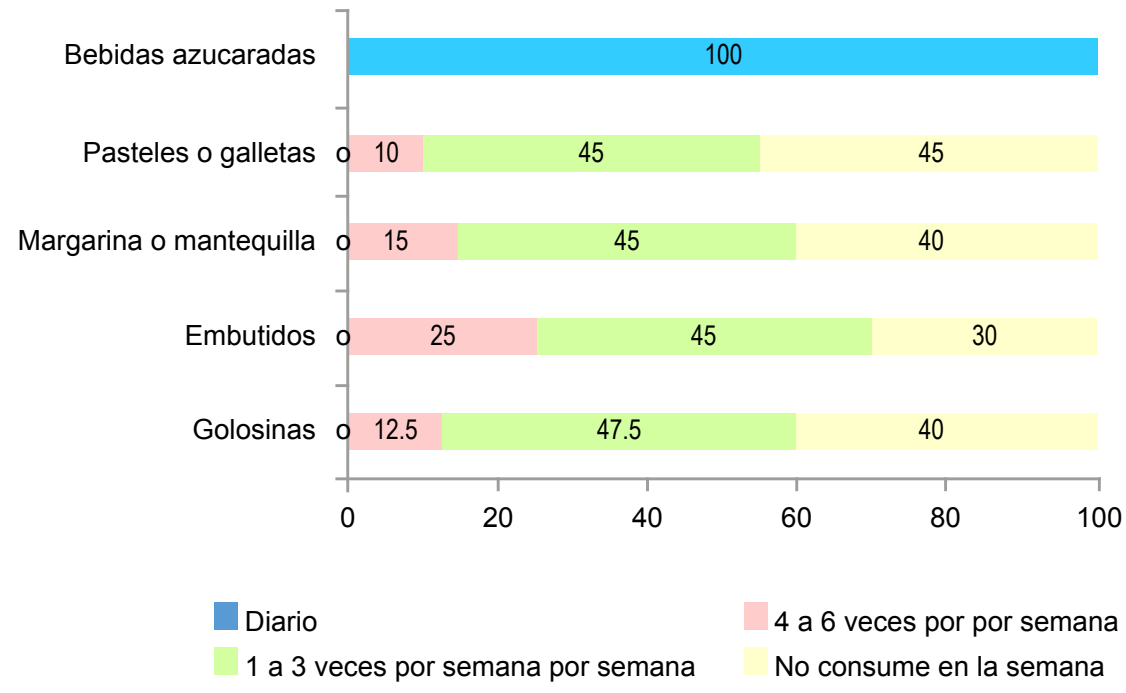

Figura 2. Frecuencia de consumo de alimentos calóricos en personas con SD 


\section{DISCUSIÓN}

El presente estudio muestra los resultados de la frecuencia de consumo de alimentos en adolescentes y adultos jóvenes con SD de tres centros de talleres privados. Aunque la muestra es pequeña, los resultados son importantes debido a que la información de los hábitos alimentarios en este grupo es escasa.

El conocimiento y análisis de los hábitos alimentarios en adolescentes y adultos jóvenes con SD es de suma importancia porque permite conocer si tienen una alimentación adecuada, ya que distintas investigaciones reportan una situación contraria ${ }^{(16,17)}$. El análisis de los hábitos alimentarios deben llevar a reflexionar sobre la necesidad de alimentarse adecuadamente y evitar malas prácticas que afecten su salud como la obesidad y otras enfermedades crónicas.

De acuerdo a las recomendaciones de la Sociedad Española de Síndrome Down ${ }^{(14)}$ y de las Guías alimentarias para la población peruana ${ }^{(15)}$, la población de nuestro estudio consume inadecuadamente pescado, huevo, verduras y hortalizas, las frutas (en la variedad y la forma de consumirlas), bebidas azucaradas pasteles o galletas, mantequilla o margarina, embutidos y golosinas.

El $70 \%$ de los participantes consumió huevo más de 4 veces por semana, este patrón es similar al que se encontró en Puno, como lo reporta Chacra (9) en su estudio de 2015. La ingesta de frutas, verduras y hortalizas fue inadecuada en nuestra investigación, este hallazgo es semejante a otro estudios realizados en San José (Costa Rica) ${ }^{(18)}$ y en la ciudad de Loja (Ecuador) ${ }^{(19)}$.

El $45 \%$ de los participantes incluyó la fruta en su alimentación diaria, este consumo es similar a que se encuentra en Pernambuco (Brasil) (20), pero, a la vez, es bastante alto respecto al consumo de frutas encontrado en Santo Domingo (Ecuador) ${ }^{(21)}$ y en Lima ${ }^{(8)}$ y Puno ${ }^{(9)}$ en Perú, donde fue menor de $40 \%$.

En relación a las frutas, la más consumida fue el plátano. El $35 \%$ de estudiantes no ingiere manzana, este rechazo se debe, probablemente, a que es una fruta muy dura y los músculos de la masticación no están lo suficientemente fortalecidos para realizar la correcta masticación. Los encuestados prefieren consumir jugos de frutas (más de 4 veces por semana) que ingerirlas picadas. Es posible que las preferencias por las frutas están en función a la masticación y deglución atípicas que los participantes del estudio pueden presentar ${ }^{(22)}$.

Respecto a los alimentos calóricos se encontró que el $45 \%$ no consumió pasteles o galletas en la semana y $40 \%$ no consumió golosinas. Estos resultados son similares a los encontrados por Farias et al. (20) donde también hallaron un reducido consumo de estos alimentos.

Se concluye que más de la mitad de los participantes tienen adecuado consumo respecto a los lácteos, carnes magras, legumbres, e inadecuado consumo respecto al pescado, huevo, verduras y hortalizas, el consumo de variedad de frutas y a la forma como consumirla, bebidas azucaradas, pasteles o galletas, mantequilla o margarina, embutidos y golosinas.

Se recomienda realizar intervenciones educativas sobre una alimentación saludable para personas con SD, en los centros donde ser realizó esta investigación, que sean dirigidas a los adolescentes y adultos jóvenes con SD, a los padres de familia, familiares y profesores.

Agradecimientos: A las instituciones que me brindaron las facilidades para la realización de este estudio. Al doctor Eduardo Maria Moreno Vivot, médico especialista en síndrome de Down y colaborador de ASDRA por sus contribuciones para la realización de este estudio. A la magister Ivonne Bernui Leo, especialista en encuestas alimentarias, por sus aportes para la realización de este estudio.

\section{REFERENCIAS BIBLIOGRÁFICAS}

1. Organización Mundial de la Salud. Día Mundial del Síndrome de Down, 21 de marzo [Internet]. 2018. Disponible en: http://www.un.org/es/events/downsyndromeday/index.shtml

2. Consejo Nacional para la Integración de la Persona con Discapacidad (CONADIS). Síndrome de Down en el Perú: Informe Temático $\mathrm{N}^{\circ} 2$ [Internet]. Lima, 2016. Disponible en: http://www.conadisperu.gob.pe/observatorio/estadisticas/ informe-estadistico-trimestral-ii-abril-junio-2016/

3. Borrel Martínez JM, Garza Morales MA, Moreno Vivat EM. Programa iberoamericano de salud para personas con síndrome de down [libro electrónico]. Madrid: FIADOWN; 2015. Disponible en: https://www.asdra.org.ar/programaiberoamericano-de-salud-para-personas-con-sindrome-dedown/

4. Moreno Vivot EM. Crecimiento y desarrollo en las personas con síndrome de Down. Rev Virtual Fundación Iberoamericana Down21. 2011 Oct. Disponible en: https://www.down21. org/revista-virtual/1066-revista-virtual-2011/revista-virtualoctubre-2011-numero-125/articulo-profesional-crecimientoy-desarrollo.html

5. Goncalves Pereira JFC. Obesidade na Síndrome de Down [Tesis]. Porto: Universidade do Porto. Faculdade de Ciencias Da Nutricao e Alimentacao; 2009.

6. Federación Española de Síndrome de Down. Programa Español de Salud para Personas con Síndrome de Down [libro electrónico]. Madrid: DOWN ESPAÑA; 2011. Disponible en:http://www.sindromedown.net/wp-content/ uploads/2014/09/90L_downsalud.pdf 
7. Faulks D, Collado V, Mazille MN, Veyrune JL, Henneguin M. Masticatory dysfunction in persons with Down's syndrome. Part 1: aetiology and incidence. J Oral Rehabil. 2008 Nov; 35(11): 854-62.

8. Gómez Hinostroza SP, Gutiérrez Mamani ME. Componente endomórfico y porcentaje de masa grasa de los adolescentes con síndrome de Down y su relación con la ingesta de energía según el nivel de actividad física 2011 [Tesis]. Lima: Universidad Nacional Mayor de San Marcos. Facultad de Medicina Humana; 2012.

9. Charca Noblega SE. Estado nutricional y consumo de alimentos de niños con Síndrome de Down en instituciones educativas de la ciudad de Puno, 2015 [Tesis]. Puno: Universidad Nacional del Altiplano. Facultad de Ciencias de la Salud; 2015.

10. Sánchez Ruíz F, De la Cruz-Mendoza F, Cereceda-Bujaico M, Espinoza-Bernardo S. Asociación de hábitos alimentarios $y$ estado nutricional con el nivel socioeconómico en adultos mayores que asisten a un Programa Municipal. An Fac Med. 2014; 75(2): 107-11.

11. Instituto de Nutrición de Centro América y Panamá. Manuel de instrumentos de evaluación dietética [libro electrónico]. Guatemala: INCAP; 2006. Disponible en: http://www.incap. int/index.php/es/publicaciones/doc_view/77-manual-deinstrumentos-de-evaluacion-dietetica.

12. Pérez Rodrigo C, Aranceta J, Salvador G, Varela-Moreiras G. Métodos de Frecuencia de consumo alimentario. Rev Esp Nutr Comunitaria. 2015; 21(1): 45-52.

13. Aparco JP, Bautista-Olórtegui W, Pillaca J. Evaluación del impacto de la intervención educativa-motivacional "como jugando" para prevenir la obesidad en escolares del Cercado de Lima: resultados al primer año. Rev Perú Med Exp Salud Pública. 2017 Jul-Set; 34(3): 386-94.

14. Federación Española de Síndrome de Down. ¡Estoy en plena forma!-Guía para familias y profesionales [libro electrórico]. Madrid: DOWN ESPAÑA; 2009. Disponible en: https://www. sindromedown.net/wp-content/uploads/2014/09/46L_ estoyen.pdf

15. Ministerio de Salud. Guías alimentarias para la población peruana [libro electrónico]. Lima: MINSA; 2018. Disponible en: https://cdn.www.gob.pe/uploads/document/file/274420/ RM_1353-2018-MINSA.PDF

16. Madrigal Loria A, González Urrutia AR. Nutritional status of children with Down sindrome from the National Center for Special Education in Costa Rica. Rev Costarric Salud Pública; 2009; 18(2): 72-8.

17. Moura Nunes A, Alves de Sousa AM, de Sousa Sá OM, Araújo Sampaio F.Diagnóstico nutricional de crianças e adolescentes com síndrome de Down em Teresina-PI. Rev Interdisciplinar. 2016; 9(4): 20-7.

18. Layne N. Relación de los hábitos alimentarios y el estado nutricional de niños y adolescentes de 6 a 17 años con síndrome de Down que residen en el gran área metropolitana, 2018 [Tesis]. San José: Universidad Hispanoamericana. Facultad de Ciencias de la Salud; 2018.

19. Cueva Loaiza AM. Prácticas alimentarias en niños y adolescentes con síndrome de Down que asisten a las escuelas especiales $\mathrm{N}^{\circ} 1,2$ y el CADE de la ciudad de Loja [Tesis]. Loja: Universidad Nacional de Loja. Facultad de la Salud Humana; 2017.

20. Farias de Queiroz M, De Santana Cirilo MA, Silva Viana MG, Cavalcante Galvao GK, Guimarães Negrmonte A, Andrade Figueiredo $M$, et al. Perfil nutricional de portadores de síndrome de Down no Agreste de Pernambuco. Nutr Clín Diet Hosp. 2016; 36(3): 122-9.

21. Freire Gómez KA, Valenzuela García BI. Análisis del estado nutricional y hábitos alimentarios en estudiantes con Síndrome de Down de la unidad educativa "Fe y Alegría" de Santo Domingo de los Tsáchilas durante el mes de Noviembre 2017 [Tesis]. Santo Domingo: Pontifica Universidad Católica del Ecuador. Facultad de Enfermería: 2017.

22. Limache Mendoza KE, Pinedo Cerrate P. Características de la masticación y deglución en niños con síndrome de Down de 6 a 9 años de una institución privada del distrito de Surco [Tesis]. Lima: Pontifica Universidad Católica del Perú. Facultad de Psicología; 2012.

Fuentes de financiamiento:

Este artículo ha sido financiado por la autora.

Conflictos de interés:

La autora declara no tener ningún conflicto de interés.

\section{Correspondencia:}

Mayra Guisela Barco Díaz

Dirección:Leoncio Prado 545 Dpto 16, Surquillo . Lima, Perú.

Teléfono: 944197525

Correo electrónico: mayraguisell@hotmail.com

\section{Recibido: 09 de enero de 2019. \\ Evaluado: 12 de febrero de 2019 Aprobado: 25 de marzo de 2019.}

(c) La revista. Publicado por Universidad de San Martín de Porres, Perú. (cc) bajo términos de Licencia Creative Commons Atribución 4.0 Internacional. (http://creativecommons.org/licenses/by/4.0/)

\section{ORCID iDs}

Mayra Guisela Barco Díaz

https://orcid.org/0000-0001-5214-0492 